\title{
Effects of bone marrow mononuclear cells on induction of axonal sprouting in cortico-cortical and cortico-striatal pathways in an animal model of cortical ablation.
}

Maria de Fatima dos Santos Sampaio ( $\nabla$ mfss.sampaio@gmail.com )

Universidade de Sao Paulo Faculdade de Medicina de Ribeirao Preto

Arthur Giraldi Guimarães

Universidade Estadual do Norte Fluminense Darcy Ribeiro

Camila da Silva Lourenço

Universidade Estadual do Norte Fluminense Darcy Ribeiro

Messias Gonzaga Pereira

Universidade Estadual do Norte Fluminense Darcy Ribeiro

Norberto Cysne Coimbra

Universidade de Sao Paulo Faculdade de Medicina de Ribeirao Preto

\section{Research note}

Keywords: bone marrow mononuclear cells, neocortical plasticity, cerebral ablation, cortico-cortical tract, cortico-striatal tract

Posted Date: May 20th, 2020

DOl: https://doi.org/10.21203/rs.3.rs-24167/v2

License: (c) (i) This work is licensed under a Creative Commons Attribution 4.0 International License.

Read Full License

Version of Record: A version of this preprint was published at BMC Research Notes on June 3rd, 2020. See the published version at https://doi.org/10.1186/s13104-020-05116-z. 


\section{Abstract}

\section{Objectives}

Many therapies have been proposed in order to investigate the mechanisms of neural repair associated with neurological diseases, including BMMC (Bone marrow Mononuclear Cells) transplantation. However, studies show that some brain injuries are less responsive to neural repair, such as, for example, cortical ablation. On the other hand, models of cortical ablation have shown functional recovery after BMMC transplantation. Thus, it is relevant to expand knowledge about BMMC, also, in the induction of neuroplasticity, in this model, considering treating a promising event for the rehabilitation of neurological diseases. Using the experimental model of cortical ablation, adult male Wistar rats, which is known to be poorly responsive to neuroplasticity, the aim of this study was to investigate the effects of BMMC on axonal sprouting induction in cortico-cortical and cortico-striatal projections. An anterograde neurotracer was used to evaluate the distribution of axonal fibers.

\section{Results}

Quantitative analyses were performed and the results showed that BMMC were not able to significantly induce axonal sprouting in the evaluated areas. Our results reinforced the idea that cortical ablation may be less responsive to neuroplasticity and the beneficial effects of BMMC therapy depend on the particularities of a neural microenvironment intrinsic to a given lesion.

\section{Introduction}

Injuries to the motor cortex or corticospinal tract interfere with motor control, making movement generation and coordination difficult and causing functional losses. [1]. Many therapies have been proposed to investigate the mechanisms of neural repair, including bone marrow mononuclear cells transplantation (BMMC).

BMMC consist of hematopoietic progenitor cells and hematopoietic stem cells, lymphocytes, monocytes and a small number of mesenchymal stem cells (MSCs). They have shown beneficial effects by presenting the ability, among others, to release trophic factors and cytokines that favour neuroprotection and repair of injured tissue and sensorimotor functional recovery [2-7].

One of the important events seen as associated with rehabilitation after CNS injury is neuroplasticity. However, it is known that this process is different for each injury [8-13].

In a previous study [14], our group investigated the effects of BMMC on functional recovery after focal cortical ablation in rats. Given the scientific evidence about neuroplasticity [15-17], we considered the instigating cortical ablation model to evaluate the efficiency of BMMC in the induction of brain plasticity. Thus, this study aimed to investigate the effects of BMMC on induction of axonal sprouting in cortico- 
cortical and cortico-striatal projections. For this, an anterograde neurotracer was used to evaluate the distribution of axonal fibers in areas of the neocortex and ipsi neostriatum and contralateral to the lesion.

\section{Methods}

\section{Animals and Surgery}

A total of 30 adult male Wistar rats (weighing $320-400 \mathrm{~g}$ ), provided by the Darcy Ribeiro Northern Fluminense State University (UENF), with 3-4 months of age. All experimental protocols were also approved by the Commission of Ethics in Animal Experimentation of UENF (process 086/2010) on $09 / 02 / 2010$. Rats were housed in groups of four per cage with food and water available ad libitum in a temperature-controlled room $\left(22 \pm 1^{\circ} \mathrm{C}\right)$ under a $12 \mathrm{~h} / 12 \mathrm{~h}$ light/dark cycle (lights on at 7:00 a.m.). Rats were transported to the experimental room in their home cages and left undisturbed for $1 \mathrm{~h}$ prior to the experiments.

Ablation was performed by cerebral cortex aspiration, as previously described [8]. Briefly, after anaesthesia with ketamine hydrochloride at $90 \mathrm{mg} / \mathrm{kg}$, i.p. (Ketamine Agener, União Química Farmacêutica Nacional, Embu-Guaçu, SP, Brazil; $0.2 \mathrm{~mL}$ of $10 \%$ solution) and xylazine hydrochloride at 10 $\mathrm{mg} / \mathrm{kg}$, i.p. (Dopaser ${ }^{\circledR}$, Hertape/Calier, Juatuba, Minas Gerais, Brazil), the left frontoparietal cortex (+2 to $-6 \mathrm{~mm}$ A.P. from the bregma) was exposed and aspirated with a pipette tip (1 ml) attached to a vacuum pump. A piece of collagen haemostatic sponge was put inside the lesion, the skin was sutured, and the animals were kept warm under a hot lamp and returned to colony room after recovery from anaesthesia.

\section{BMMC obtaining and transplantation}

Bone marrows were obtained from donor naïve rats $(n=3)$, as previously described [2]. The animals were euthanized with a lethal dose of ketamine hydrochloride (i.p.) and xylazine hydrochloride (i.p.). BMMCs were collected and washed with phosphate-buffered saline (PBS). Following cell count, they were resuspended in sterile physiological saline, and the final concentration was approximately $3 \times 10^{7}$ BMMCs $/ 0.5 \mathrm{~mL}$.

Twenty four hours after surgical procedure, animals were anaesthetised with ketamine hydrochloride (90 $\mathrm{mg} / \mathrm{kg}$, i.p.) and xylazine hydrochloride (10 mg/kg, i.p.) and injected through the left jugular vein with $B M M C$ or vehicle (PBS). The skin was sutured, and the animals were kept warm under a hot lamp and returned to colony room after recovery from anaesthesia.

\section{Experimental design}


The animals were randomly divided into experimental groups. NAÏVE (animals without lesion), CONT (animals submitted to cortical ablation and treated with vehicle), BMMC (animals submitted to cortical ablation and treated with mononuclear cells of bone marrow). The therapeutic window was chosen based on our previous studies [5].

Animals from the NAIIVE $(n=7)$, CONT $(n=6)$ and BMMC $(n=9)$ groups were subjected to anterograde tracer microinjections in sixty days after the surgical procedure. In all groups, the animals were euthanized 8 days after the tracer microinjections.

\section{Characterisation of the lesion}

For the characterisation of the lesion, four untreated animals were euthanised $72 \mathrm{~h}$ after cortical ablation with a lethal dose of ketamine hydrochloride (i.p.) and xylazine hydrochloride (i.p.). The brain was rapidly, according Sampaio et al [5]. The slices were immersed for 30 min into 2\% 2,3,5triphenyltetrazolium chloride (TTC, T8877/1 Sigma-Aldrich) solution at $37^{\circ} \mathrm{C}$. Digital images were captured from reacted slices with a camera coupled to a dissecting microscope and to a computer.

\section{In vivo neural tract tracing procedure}

Sixty days after cortical ablation, the animals were anaesthetised with xylazine hydrochloride $(10 \mathrm{mg} /$ $\mathrm{kg}$, i.p.) and ketamine hydrochloride ( $90 \mathrm{mg} / \mathrm{kg}$, i.p.), and fixed in a stereotaxic apparatus. A small trepanation was performed in the calvaria over the sensorimotor cortex in the hemisphere contralateral to the lesion. Using a stereotaxic coordinates obtained from a rat brain atlas (anteroposterior axis, $+1.2 \mathrm{~mm}$, mid-lateral axis, $-2.7 \mathrm{~mm}$ ), the $10 \%$ AlexaFluor 546-conjugated biodextran amine (BDA) solution (Dextran, Alexa Fluor 546; 10,000 MW, Anionic, Fixable, Life Technologies, Austin TX, USA) was microinjected into the primary motor cortex (M1) through a Hamilton syringe. The injections were made in the depths of $1.8 \mathrm{~mm}$ and $2.0 \mathrm{~mm}$ from Bregma, with a total volume of solution injected of $0.5 \mu \mathrm{L}$.

\section{Histological analysis}

After 8 days of the neural tract tracer injection, each animal was euthanized with a lethal dose of with ketamine hydrochloride (i.p.) and xylazine hydrochloride (i.p.). Immediately after this procedure, the encephalon was perfused through the left ventricle with physiological saline solution $(\mathrm{NaCl}, 0.9 \%)$, followed by $4 \%$ paraformaldehyde solution in phosphate buffer $(0.2 \mathrm{M}, \mathrm{pH} 7.4)$. The encephalon was rapidly removed and incubated in $20 \%$ sucrose dissolved in PBS $(0.2 \mathrm{M}, \mathrm{pH} 7.4)$ for 24 hours, soaked in a compound (Tissue Tek O.C.T.) and immersed in liquid nitrogen for freezing. It was then sectioned in the coronal plane ( $30 \mu \mathrm{m}$ thick), at $-20^{\circ} \mathrm{C}$ in a cryostat (CM 1950 Leica, CM 1950 Leica, Wetzlar, Germany) 
and the slices arranged in gelatinised slides, which were subsequently visualised under a motorised fluorescence microscope (Axiolmager Z1 with APOTOME Il; Zeiss, Oberkochen, Germany).

\section{Data analyses}

The images were selected and treated using the ImageJ software $(\mathrm{NIH})$. From the efficacy of the neural tract tracing, in each two brain sections slides per animal, 3 fields were analysed as follow: Neocortex surrounding the lesioned area and the neostriatum ipsilateral and contralateral to the cortical brain lesion (figure $2 \mathrm{~J}$ ). The mean per animal was calculated in respective areas and then the mean for each experimental group was plotted for the statistical analyses. The percentage of axonal fibres was computed based on the number of axons identified per unit of visual field area.

Data from the experiment were submitted to the one-way ANOVA, followed by Tukey's post hoc test. Values were reported as the mean and standard error of the mean (S.E.M.). P-values $<0.05$ were considered statistically significant. The distribution of the variables passed in the normality test (ShapiroWilk normality test).

\section{Results}

\section{Histochemical characterisation of the cortical lesion}

Using TTC, it was demonstrated that all lesioned animals, $72 \mathrm{~h}$ after each the surgical procedure, showed in the sensory-motor region, loss of neocortical tissue restricted to the sensory-motor cortical area when submitted to the cortical ablation, as shown in figure 1.

Figure 1 - Extension of the ischemic lesion induced by ablation

\section{Quantitative analysis of cortico-cortical and cortico-striatal projections of naive rats and those submitted to a unilateral cortical ablation after BMMC or vehicle transplantation}

Statistical analysis was performed exclusively with data from the animals that presented evidence that microinjection in the primary motor cortex (M1) was successfully performed.

Results of quantitative morphological analysis showed that intravenous treatment with BMMC did not significantly increase the number of BDA-positive axonal fibers in areas of the neocortex and ipsilateral neostriatum and contralateral to the lesion (figure 2A - I). Analysis of the neural tissue ipsilateral to the cortical lesion and surrounding the brain damage, according to the one-way ANOVA, there was a significant effect of the treatment $\left(F_{2,16}=3.992, p<0.05\right)$. The neocortical lesion with cortical tissue ablation of animals not treated with BMMC (CONT group) was followed by a significant decrease 
in the number of BDA-labeled axonal fibers in the cortical layers surrounding brain injury compared to healthy rodents (NAÏVE group) ( Tukey post hoc test; $p<0.05$ ). These findings are shown in Fig. $2 \mathrm{~K}$.

Regarding the the neostriatum ipsilateral to the brain lesion, there was a significant effect of the treatment $\left(F_{2,13}=6.444 ; p<0.05\right)$, according to one-way ANOVA. Control injured animals (CONT) showed a reduction in BDA-positive axonal fibers when compared to the healthy group (NAïVE group). These data are shown in Fig. $2 \mathrm{~L}$.

With respect to the caudate-putamen contralateral to the lesion, according to one-way ANOVA, there was any significant difference between the experimental groups $\left(F_{2,17}=1.143 ; p>0.05\right)$, as shown in Fig. 2M.

Figure 2 - Effect of intravenous BMMC transplantation on the number of axonal fibers of the corticocortical and cortico-striatal pathways after cortical ablation.

\section{Discussion}

Our results showed that BMMC treatment did not induce an increase in axonal fibers in areas of the contralateral cortex and ipsilateral and contralateral neostriatal lesion, of animals injured by cortical ablation. There are few studies in the scientific literature regarding cell therapy in cortical ablation models, possibly because it represents a less frequent clinical situation when compared to stroke or head trauma. Cortical ablation resembles a condition of surgical removal of a brain tumour [14].

In approaches to neuroplasticity after injury, there is scientific evidence that cortical ablation has been shown to be poorly responsive to structural plasticity [9-11]. Therefore, it became an attractive model for this work, which aimed to extend the studies of BMMC efficiency, now contemplating the induction of neuronal circuit remodelling, in an attempt to better elucidate the relationship of neuroplasticity and motor functional recovery.

The results of this study corroborate the findings in the literature $[11,18]$ showing that after cortical ablation in a sensorimotor area there was little responsiveness to cortico-cortical and cortico-striatal neuroplasticity. In this study, we also observed that treatment with BMMC was not able to reverse this picture.

Carmichael and Chesselet [19] contributed to these findings, that different sensorimotor cortex lesions may promote different responses in the induction of axonal sprouting, even being injuries of the same location and extension. These authors, using ablation and ischemia models, showed that there was an increase in axonal marking in the medial cortex and striatum, both ipsilaterally to the lesion, but only in animals submitted to ischemia. Thus, attention was paid to a brain reorganization dependent on the type of injury induced in rats, reinforcing the previous results of Szele et al [8] who indicated that aspiration lesions promoted a greater inhibition of structural plasticity, unlike cortical ischemic lesions. 
According to Freitas et al. [13], one of the possible explanations of not observing a significant response to structural plasticity after cortical ablation is due to the injury mechanism itself. Probably pathologies such as stroke are more responsive to this event, as it speeds up substantial necrosis and favours a significant inflammatory response $[11,18,20,21]$.

With regard to therapy, the results pointed out by Michael Chop reinforce that cell therapy with bone marrow stromal cells (MSCs) is a promising strategy in the induction of neuroplasticity and in functional sensorimotor recovery after ischemia [22,23], however, the model and treatment differ from this work.

Research has shown that BMMC has the capacity for neuroprotection and neural repair, but it is essential to seek therapeutic strategies that interact positively with the biological processes, inherent to each pathological process.

\section{Limitation}

The limitation of this study is to present, only, results of the treatment of BMMC in the induction of axonal sprouting, in the cortical ablation model, without comparisons with an ischemic group. However, it was considered that the cortical ablation model is described as not very responsive to plasticity, whereas, in ischemic models, treatment with cells derived from bone marrow promoted significant results.

\section{Declarations}

\section{Availability of data and materials}

Data supporting our finding are available from the corresponding author.

\section{Abbreviations}

ANOVA: Analysis of variance

BDA: Biodextran amine

BMMC: Bone marrow mononuclear cells

CEUA: Commission of Ethics in Animal Experimentation

CNS: Central nervous system

M1: Primary motor cortex

MSCs: mesenchymal stem cells

PBS: Phosphate buffered saline 
TTC: 2,3,5-triphenyltetrazolium chloride

\section{Ethics Declarations}

\section{Ethics approval and consent to participate}

All experimental protocols were also approved by the Commission of Ethics in Animal Experimentation (CEUA) of Darcy Ribeiro Northern Fluminense State University, process $086 / 2010$ on 09/02/2010 and process $151 / 2012$ on $24 / 04 / 2012$.

\section{Consent for publication}

"Not applicable" in this section.

\section{Competing interests}

The authors declare that they have no competing interests"

\section{Funding}

This research was supported by Fundação de Amparo a Pesquisa do Estado do Rio de Janeiro (FAPERJ) (grants E-26/102.179/2013), Fundação de Amparo à Pesquisa do Estado de São Paulo (FAPESP) (grants 2017/11855-8) and Conselho Nacional de Pesquisa e Desenvolvimento Tecnológico (CNPq) (grants 474853/2013-6). N.C. Coimbra is a researcher (level 1A) at CNPq (processes 301905/2010-0 and $301341 / 215-0)$.

\section{Authors' contributions}

Maria de Fátima dos Santos Sampaio performed the experiments, analysed and interpreted the data, performed the statistical analysis, designed the figures and wrote the manuscript. Arthur Giraldi Guimarães designed the experiments. Camila da Silva Lourenço performed the experiments. Messias Gonzaga Pereira performed the statistical analysis and reagents. Norberto Cysne Coimbra designed the experiments, analysed and interpreted the data, wrote the manuscript. All authors have approved the final version of the manuscript. 


\section{Corresponding author:}

Dr. Maria de Fátima dos Santos Sampaio (mfss.sampaio@gmail.com). Laboratory of Neuroanatomy and Neuropsychobiology, Department of Pharmacology, Ribeirão Preto Medical School of the University of São Paulo (FMRP-USP), Av Bandeirantes, 3900, Ribeirão Preto, 14049-900, São Paulo, Brazil.

\section{Acknowledgements}

"Not applicable" in this section.

\section{References}

[1] T.H. Murphy, D. Corbett, Plasticity during stroke recovery: from synapse to behaviour., Nat. Rev. Neurosci. 10 (2009) 861-72. doi:10.1038/nrn2735.

[2] A. Giraldi-Guimarães, M. Rezende-Lima, F.P. Bruno, R. Mendez-Otero, Treatment with bone marrow mononuclear cells induces functional recovery and decreases neurodegeneration after sensorimotor cortical ischemia in rats, Brain Res. 1266 (2009) 108-120. doi:10.1016/j.brainres.2009.01.062.

[3] C. Zaverucha-Do-Valle, L. Mesentier-Louro, F. Gubert, N. Mortari, A.B. Padilha, B.D. Paredes, A. Mencalha, E. Abdelhay, C. Teixeira, F.G.M. Ferreira, F. Tovar-Moll, S.A. Lopes De Souza, B. Gutfilen, R. Mendez-Otero, M.F. Santiago, Sustained effect of bone marrow mononuclear cell therapy in axonal regeneration in a model of optic nerve crush, Brain Res. (2014). doi:10.1016/j.brainres.2014.08.070.

[4] F.S. Vahidy, M.H. Rahbar, H. Zhu, P.J. Rowan, A.B. Bambhroliya, S.I. Savitz, Systematic Review and Meta-Analysis of Bone Marrow-Derived Mononuclear Cells in Animal Models of Ischemic Stroke, Stroke. 47 (2016) 1632-1639. doi:10.1161/STROKEAHA.116.012701.

[5] M. De Fátima Dos Santos Sampaio, F.D.S. Marcilio, A. Giraldi-Guimarães, Does treatment with bone marrow mononuclear cells recover skilled motor function after focal cortical ischemia? Analysis with a forelimb skilled motor task in rats, Brain Res. 1492 (2013) 130-139. doi:10.1016/j.brainres.2012.11.026.

[6] F. Gubert, I. Bonacossa-Pereira, A.B. Decotelli, M. Furtado, A. Vasconcelos-dos-Santos, R. MendezOtero, M.F. Santiago, Bone-marrow mononuclear cell therapy in a mouse model of amyotrophic lateral sclerosis: Functional outcomes from different administration routes, Brain Res. 1712 (2019) 73-81. doi:10.1016/j.brainres.2019.02.003.

[7] A. de Vasconcelos dos Santos, J. da Costa Reis, B. Diaz Paredes, L. Moraes, Jasmin, A. GiraldiGuimarães, R. Mendez-Otero, Therapeutic window for treatment of cortical ischemia with bone marrowderived cells in rats, Brain Res. 1306 (2010) 149-158. doi:10.1016/j.brainres.2009.09.094. 
[8] F.G. Szele, C. Alexander, M.F. Chesselet, Expression of molecules associated with neuronal plasticity in the striatum after aspiration and thermocoagulatory lesions of the cerebral cortex in adult rats., J. Neurosci. 15 (1995) 4429-48. http://www.ncbi.nlm.nih.gov/pubmed/7790918 (accessed August 27, 2019).

[9] J.A. Napieralski, R.J.A. Banks, M.F. Chesselet, Motor and somatosensory deficits following uni- and bilateral lesions of the cortex induced by aspiration or thermocoagulation in the adult rat, Exp. Neurol. 154 (1998) 80-88. doi:10.1006/exnr.1998.6893.

[10] K. Uryu, L. MacKenzie, M.F. Chesselet, Ultrastructural evidence for differential axonal sprouting in the striatum after thermocoagulatory and aspiration lesions of the cerebral cortex in adult rats, Neuroscience. 105 (2001) 307-316. doi:10.1016/S0306-4522(01)00203-2.

[11] S.T. Carmichael, Plasticity of cortical projections after stroke., Neuroscientist. 9 (2003) 64-75. doi:10.1177/1073858402239592.

[12] A. Giraldi-Guimarães, H.T. De Freitas, B.D.P. Coelho, H. MacEdo-Ramos, R. Mendez-Otero, L.A. Cavalcante, W. Baetas-Da-Cruz, Bone marrow mononuclear cells and mannose receptor expression in focal cortical ischemia, Brain Res. 1452 (2012) 173-184. doi:10.1016/j.brainres.2012.03.002.

[13] H.T. De Freitas, M.G. Rebel, B.P. Coelho, V.G. Da Silva, G.G.C. Galaxe-Almeida, A. Giraldi-Guimarães, Effect of the treatment of focal brain ablation in rat with bone marrow mesenchymal stromal cells on sensorimotor recovery and cytokine production, J. Neurol. Sci. (2015). doi:10.1016/j.jns.2014.11.032.

[14] H.T. de Freitas, V.G. da Silva, A. Giraldi-Guimarães, Comparative study between bone marrow mononuclear fraction and mesenchymal stem cells treatment in sensorimotor recovery after focal cortical ablation in rats, Behav. Brain Funct. 8 (2012). doi:10.1186/1744-9081-8-58.

[15] A. Pascual-Leone, A. Amedi, F. Fregni, L.B. Merabet, THE PLASTIC HUMAN BRAIN CORTEX, Annu. Rev. Neurosci. 28 (2005) 377-401. doi:10.1146/annurev.neuro.27.070203.144216.

[16] M. Hübener, T. Bonhoeffer, Neuronal plasticity: beyond the critical period., Cell. 159 (2014) 727-37. doi:10.1016/j.cell.2014.10.035.

[17] J.J. Overman, S.T. Carmichael, Plasticity in the injured brain: More than molecules matter, Neuroscientist. 20 (2014) 15-28. doi:10.1177/1073858413491146.

[18] A.C. Voorhies, T.A. Jones, The behavioral and dendritic growth effects of focal sensorimotor cortical damage depend on the method of lesion induction., Behav. Brain Res. 133 (2002) 237-46. doi:10.1016/s0166-4328(02)00029-3.

[19] S.T. Carmichael, M.-F. Chesselet, Synchronous neuronal activity is a signal for axonal sprouting after cortical lesions in the adult., J. Neurosci. 22 (2002) 6062-70. doi:10.1523/JNEUROSCI.22-1406062.2002 . 
[20] C.L.R. Gonzalez, B. Kolb, A comparison of different models of stroke on behaviour and brain morphology, Eur. J. Neurosci. 18 (2003) 1950-1962. doi:10.1046/j.1460-9568.2003.02928.x.

[21] L.I. Benowitz, P.G. Popovich, Inflammation and axon regeneration, Curr. Opin. Neurol. 24 (2011) 577-583. doi:10.1097/WCO.0b013e32834c208d.

[22] Xin, H, Li Y, Liu Z, Wang X, Shang X, Cui Y, Zhang ZG, Chopp M..MiR-133b Promotes Neural Plasticity and Functional Recovery After Treatment of Stroke with Multipotent Mesenchymal Stromal Cells in Rats Via Transfer of Exosome-Enriched Extracellular Particles, Stem Cells. 31 (2013) 2737-46. doi: 10.1002/stem.1409.

[23] Ding X1, Li Y, Liu Z, Zhang J, Cui Y, Chen X, Chopp M.The Sonic Hedgehog Pathway Mediates Brain Plasticity and Subsequent Functional Recovery After Bone Marrow Stromal Cell Treatment of Stroke in Mice, J Cereb Blood Flow Metab. 33(2013)1015-24. doi: 10.1038/jcbfm.2013.50.

\section{Figures}
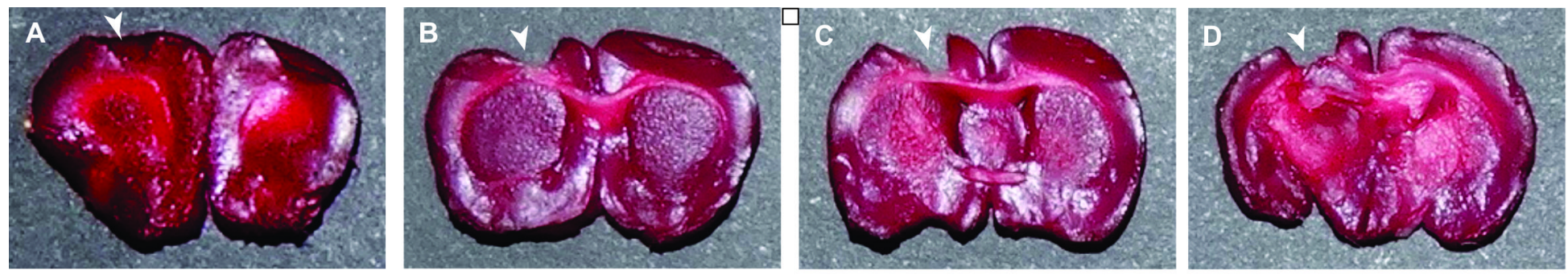

Rostral

Caudal

\section{Figure 1}

Extension of the cortical ablation procedures. The figure shows sequential coronal sections $(2 \mathrm{~mm})$ of Wistar rat brains 72 hours after each cortical lesion, submitted to the histochemical reaction with 2,3,5triphenyltetrazolium chloride (TTC), which reddens the viable tissue. (A - D) Histological sections of a brain reacted after ablation, observing the removal of the sensorimotor area (white arrowheads in the top). 

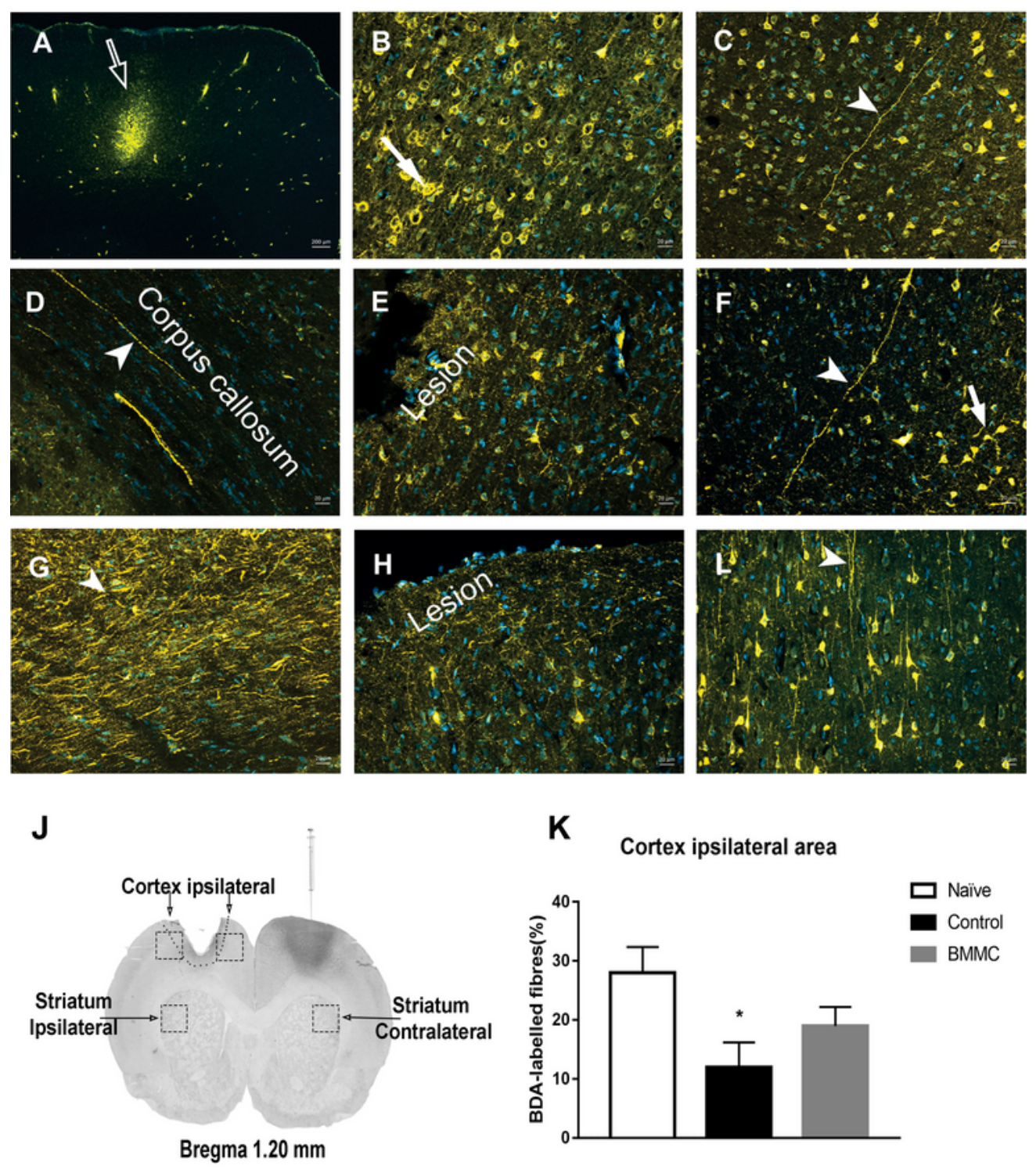

L Striatum ipsilateral area

M Striatum contralateral area
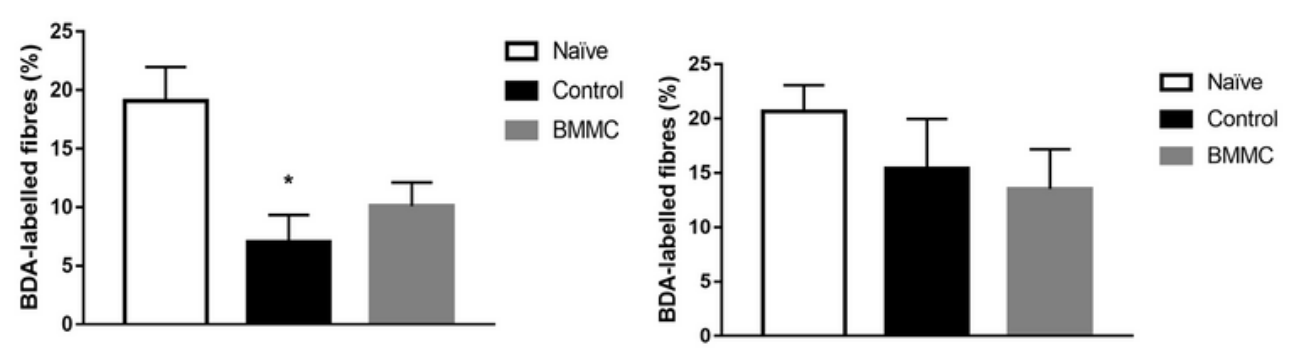

\section{Figure 2}

Photomicrographs of transverse sections of "NAÏVE" Wistar rats brain (A-C), of "CONT " Wistar rats brain (D-F), of "BMMC " Wistar rats brain (G-I). Representative site of injection of the anterograde AlexaFluor 546-conjugated biodextran amine (BDA) neurotracer in the primary motor cortex (A), fibres of passage in the external pyramidal layer $(B)$, corpus callosum to connect the contralateral ( $D$ and $G)$, cerebral cortex ipsilateral to lesion (E), internal pyramidal layer $(\mathrm{H})$ and fusiform layer. $(\mathrm{C}, \mathrm{F}$ and I). Representative site of 
injection scheme of the anterograde AlexaFluor 546-conjugated biodextran amine (BDA) neurotracer in the primary motor cortex, demonstrating the quantified areas for analysis of cortico-cortical and corticostriatal projections $(\mathrm{J})$. The effect of the transplantation of BMMC or vehicle-treatment on axonal sprouting in cortical layers surrounding the brain lesions $(K)$ and in the neostriatum ipsilateral $(L)$ and contralateral $(\mathrm{M})$ to the cortical ablation were also demonstrated. Data were represented as mean $\square$ S.E.M. ( $n=6-9$ rats per group). ANOVA (one-way) followed by Tukey's post hoc test. ${ }^{*} p<0.05$, in comparison to the NAIIVE group. Arrowhead indicate BDA-labelled neuronal fibres, transparent arrow indicate site of injection of the anterograde AlexaFluor 546-conjugated biodextan amine (BDA) neurotracer in the primary motor cortex (M1) and white arrows indicate BDA-labelled perikarya located in different brain structures. Scale bars: $200 \square \mathrm{m}$ on the panel A; $20 \square \mathrm{m}$ on the panels B, C, D, E, F, G, H and I.

\section{Supplementary Files}

This is a list of supplementary files associated with this preprint. Click to download.

- NC3RsARRIVEGuidelinesChecklist2014.docx 